\title{
Aufsätze
}

\section{Andreas Fisahn Griechenland und die Perspektiven der EU}

Georg Soros, Autor und Fondsmanager, was eine Untertreibung seiner Funktion ist, schlägt der deutschen Regierung ein Gedankenexperiment vor: Sie solle sich vorstellen, Deutschland verabschiede sich von der gemeinsamen Währung und führe die Deutsche Mark wieder ein. Absehbare Folgen wären eine steile Aufwertung der Mark, ein Sinken der Konkurrenzfähigkeit gegenüber den anderen EU-Staaten, Sinken der Exporte, Steigen der Arbeitslosigkeit usw. - dafür könnten deutsche Rentner in Spanien leben wie Gott in Frankreich; ${ }^{1}$ kurz: Deutschland ist der Hauptprofiteur des Euro, weil es im Produktivitätsvergleich im EuroRaum eine unterbewertete Währung hat, die es erlaubt, Vizeexportweltmeister hinter China zu sein. Das Wissen um diese Situation müsste die Bundesregierung veranlassen, pfleglich mit den europäischen Nachbarn umzugehen, sich in großer europäischer Solidarität zu üben. Weil dem nicht so ist, weil Deutschland Sparen zum obersten Ziel für sich und die anderen Mitgliedstaaten erklärt, weil es eine Wirtschaftsregierung für den Euro-Raum ablehnt, lautete die Überschrift über dem Kommentar von Soros: „Deutschland schadet der EU“. Das ist aber nur die halbe Wahrheit. Die "Griechenlandkrise" hat tiefer liegende Probleme und strukturelle Mängel der Konzeptionierung des Euro und der EU sichtbar werden lassen.

\section{Die griechische Tragödie}

Der erste Akt der griechischen Tragödie fand schon Anfang 2010 statt. „Spiegelonline“ titelte am 3. Februar 2010: „EU-Sparkommissar Almunia diktiert Griechenland den Kürzungskurs. Europa verliert die Geduld mit Griechenland - und verordnet dem Krisenstaat ein hartes Kürzungsprogramm. EU-Währungskommissar Almunia erwartet nun regelmäßige Sparberichte." Die EU-Kommission hatte den Sanktionsmechanismus bei übermäßigem Defizit gemäß Art. 126 AEUV in Gang gesetzt und für Griechenland „Empfehlungen“ ausgesprochen, die exakt dem entsprechen, was im Mai der Welt als griechisches Sparpaket präsentiert wurde. Die Zustimmung zum „Rettungspaket für Griechenland“ war entgegen dem öffentlichen Anschein nicht das Ergebnis des vorherigen Ringens um eine Konsolidierung des griechischen Haushalts oder - so sähe sie es gern des Drucks und der Standfestigkeit der deutschen Regierung.

Das Verfahren bei übermäßigem Defizit wird eingeleitet, wenn die sog. Maastricht-Kriterien verletzt sind, die gebieten, dass die Neuverschuldung eines Staates $3 \%$ seines Bruttoinlandsprodukts (BIP) und die Gesamtverschuldung, das ist der addierte Schuldenberg, $60 \%$ des BIP nicht übersteigen dürfen. Griechenland liegt in beiden Werten deutlich darüber. Die Finanz- und Wirtschaftskrise hat allerdings dazu geführt, dass 2010 vermutlich kein Euroland unter den $3 \%$ bleibt. 
Für den Euro-Raum wurde für 2009 eine Gesamtverschuldung von 78,7 \% des BIP errechnet, für 2010 prognostiziert, dass die Quote bei 84,7 \% liegt und im folgenden Jahr auf 88,5 \% steigt. ${ }^{2}$ Deutschland soll dieses Jahr bei 78,8 \% Gesamtverschuldung liegen. Nun könnte man ausrechnen, wie hoch das Wirtschaftswachstum sein muss, um selbst bei illusorischen $0 \%$ Neuverschuldung unter die 60 \% zu kommen. Aus ökologischen Gründen wäre ein solches Wachstum aber keineswegs wünschenswert. Das ist das erste Dilemma nicht nur der europäischen Politik, vielmehr des kapitalistischen Systems insgesamt.

Im zweiten Akt des griechischen Dramas, das im März 2010 aufgeführt wurde, erhielt Griechenland das Versprechen von der EU, dem IWF und von den Mitgliedstaaten, Finanzhilfen zu erhalten. Dabei versicherten die Regierungschefs: „Das Ziel dieses Mechanismus wird nicht darin bestehen, Finanzmittel zu durchschnittlichen Zinssätzen des Euro-Währungsgebiets bereitzustellen, sondern darin, Anreize für eine schnellstmögliche Rückkehr an den Finanzmarkt zu risikogerechten Preisen zu setzen. Die Zinssätze werden nicht konzessionär sein, d.h. sie werden kein Subventionselement enthalten. Beschlüsse im Rahmen dieses Mechanismus werden in voller Übereinstimmung mit dem Vertragsrahmen und

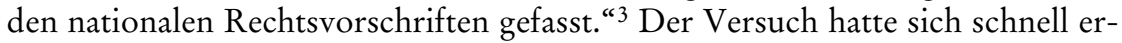
ledigt, die Zinsen für griechische Staatsanleihen stiegen weiter.

Das Rettungspaket bestand im dritten Akt aus zwei wesentlichen Elementen: den Bürgschaften und Kreditzusagen der EU und den Mitgliedstaaten sowie den Staatsanleihen seitens der Europäischen Zentralbank (EZB). Am 10. Mai 2010 beschloss der ECOFIN ${ }^{4}$ ein Maßnahmenprogramm, das in der schönen Sprache der europäischen Public Relations als „Europäischer Stabilitätsmechanismus“ bezeichnet wird. Das Paket hat eine Größenordnung von 500 Mrd. Euro, die von der EU aufgebracht werden, und weiteren $250 \mathrm{Mrd}$. Euro, die vom IWF bereit gestellt werden sollen. Die EU gewährt Griechenland danach Kredite in Höhe von 60 Mrd. Euro. Weitere 440 Mrd. Euro sollen über den Kapitalmarkt aufgenommen und von den Mitgliedstaaten garantiert werden. Der IWF gewährt zu den gleichen Konditionen wie die EU weitere Kredite in Höhe von $250 \mathrm{Mrd}$. Euro an Griechenland. ${ }^{5} \mathrm{Ob}$ das Paket die erhofften Wirkungen hat, ist fraglich. Nach dem Beschluss sank die Rendite der griechischen Staatsanleihen von fast zehn Prozentpunkten Aufschlag auf Bundesanleihen auf etwas über vier Prozentpunkte Aufschlag. Inzwischen, Ende Juni 2010, verlangt „der Markt “6 wieder einen Aufschlag von fast acht Prozentpunkten. ${ }^{7}$

\section{Solidarität und Eigennutz}

Die Bundesregierung haderte mit dem Rettungspaket, weil es gegen EU-Recht verstoße, die Misswirtschaft der Griechen noch belohne oder auch nur, weil Wahlen anstanden und die in der Selbstwahrnehmung fleißigen Deutschen nicht

2 European Economic Forecast Spring 2010, S. 16; http://ec.europa.eu/economy_finance/ publications/ european_economy/2010/pdf/ee-2010-2_en.pdf.

3 Erklärung der Staats- und Regierungschefs der Mitgliedstaaten des Euro-Währungsgebietes vom 25.3.2010.

4 Als Ecofin-Rat (auch EcoFin oder ECOFIN) wird der Rat der Europäischen Union in der Zusammensetzung „Wirtschaft und Finanzen“ bezeichnet. Dem Rat gehören die Wirtschafts- und Finanzminister der EU-Mitgliedstaaten an. Er tagt in der Regel einmal im Monat.

5 Pressemitteilung zur außerordentlichen Sitzung des ECOFIN am 9./10.5.2010, http://www.consilium.europa.eu/uedocs/cms_data/docs/pressdata/en/ecofin/114324.pdf.

6 FTD 28.6.2010, S. 21.

7 Gleichzeitig haben die Ratingagenturen damit begonnen, die Schulden des Landes auf Ramschniveau herunterzustufen. Das heißt, die eigentliche Intention des Paketes, eine „Marktberuhigung herbeizuführen, ohne dass die Mittel in Anspruch genommen werden“, scheint gescheitert zu sein. Bei einem neuerlichen Refinanzierungsbedarf wird Griechenland folglich die Mittel aus dem Paket in Anspruch nehmen müssen. 
nur an den Stammtischen über die „Sirtaki-Mentalität" ${ }^{\text {“8 }}$ herzogen. Dieses Zaudern trieb den Preis für die Rettung Griechenlands oder des Euros gewaltig in die Höhe. Schließlich hat sich die Bundesregierung beteiligt - angeblich, weil die Griechen vorher ein rigides Spar- und Konsolidierungsprogramm verabschiedet hatten. Tatsächlich dürfte sich in der Bundesregierung die Erkenntnis durchgesetzt haben, dass es nicht nur um Griechenland geht. Abgesehen von der Möglichkeit, dass Zinsspekulationen auch gegen andere Staaten befürchtet wurden und diese auch in den Strudel der Insolvenz hätten geraten können, dient der „Stabilisierungsmechanismus“ der EU vor allem der Stabilisierung der Kapitalmärkte oder besser der Banken. Da die nordeuropäischen Banken mehr oder weniger stark in Griechenland (und den anderen südlichen Euro-Ländern) engagiert sind, ${ }^{9}$ war zu befürchten, dass ein griechischer Staatsbankrott sich mindestens auf den Kreditmarkt im restlichen Europa auswirkt und wieder zu der gefürchteten Kreditklemme führen könnte.

Aus der Krise 2008 hätte man lernen können, dass es wichtig ist, rechtliche Instrumentarien zu schaffen, um die Banken oder die „Finanzindustrie“ durch Mechanismen der Umschuldung an den Kosten von drohenden Insolvenzen und Krisen zu beteiligen. Eine Überschuldung hat immer mindestens zwei Partner, den Schuldner und den Gläubiger, der offenbar zu leichtfertig Kredite eingeräumt hat. Statt dessen wurde auch in der Griechenlandkrise mit Blick auf den Rettungsschirm das Motto autoritären Denkens ausgegeben: „There is no Alternative“. Die scheinbare Solidarität war letztlich „deutscher"10 Eigennutz.

\section{Das zweite Dilemma der Verschuldenskrise}

Seitdem geriert sich die Bundesregierung als Zerberus der Preisstabilität, der darüber wachen will, dass die Euro-Länder nicht nur die Maastricht-Kriterien einhalten, sondern in ihre Verfassung nach deutschem Vorbild eine Verschuldensbremse einbauen sollen, deren Wirkung keineswegs erwiesen ist - weniger vorsichtig formuliert: die für die Schuldenentwicklung vermutlich die gleiche Bedeutung haben wird wie die Alchemisten für die Staatshaushalte der frühen Neuzeit. Dabei ist der ausschließliche Blick auf die Verschuldung eines Landes höchst unzureichend. Das macht ein Vergleich zwischen der Situation Japans und Griechenlands sichtbar.

Der IWF geht davon aus, dass die Staatsschulden Japans 2010 auf 227 Prozent des Bruttoinlandsprodukts (BIP) steigen. Für 2014 rechnet er mit 246 Prozent. Griechenland wird geschätzt auf eine Gesamtverschuldung von 124,9\% in 2010 und 135,4 \% des BIP in 2011. ${ }^{11}$ Im Unterschied zu Griechenland bestand bisher nicht die Gefahr, dass Japan wegen dieser hohen Schuldenquote eine Staatspleite anmelden muss. Offenbar sind andere Faktoren in die Betrachtung der ökonomischen Situation einzustellen. Dazu gehören die Steuerquote, die Leistungsbilanz oder die Sparquote. Die zehnjährigen japanischen Staatsanleihen werden mit nur 1,x Prozent verzinst, dementsprechend gering ist die Zinslast der Regie-

8 Ernak Ferlemann, Staatssekretär im Bundesverkehrsministerium, erklärte beispielsweise, dass die „armen Griechen ... ja in den letzten Jahren wahrscheinlich mehr Sirtaki getanzt als gearbeitet haben“ (Weser Kurier v. 4.5.2010).

9 Nach: http://www.jjahnke.net/fb.html.

10 Einen einheitlichen nationalen Eigennutz gibt es in einer differenzierten Gesellschaft nicht, es nützt primär den Banken und „letztlich auch“ - wie Frau Merkel es so schön formulierte - auch dem Bürger.

11 Bundesministerium der Finanzen, Monatsbericht des BMF, Dezember 2009, http://www.bundesfinanzministerium.de/nn 53848/DE/BMF_Startseite/Aktuelles/Monatsbericht des_BMF/2009/12/ inhalt/Monatsbericht-Dezember-2009, property=publicationFile.pdf. 
rung. ${ }^{12}$ Ein Grund liegt darin, dass Japan sich im Wesentlichen bei sich selbst und nicht im Ausland verschuldet hat. 53 \% der 937000 Mrd. Yen (7540 Mrd. Euro) an ausstehenden Anleihen werden vom öffentlichen Sektor selbst gehalten. Auch Private investieren vorzugsweise in japanische Anleihen und können dies bei in der Krise zwar reduzierter, aber bislang positiver Sparquote. ${ }^{13}$ Hier liegt ein Unterschied zu Griechenland, dessen Bürger mit Blick auf die Sparquote im negativen Saldo liegen, das sich deshalb im Wesentlichen im Ausland verschuldet hat. Die „vom Markt“ geforderten Zinsen für griechische Staatsanleihen kletterten so vor dem 750 Mrd. Euro-Paket auf über zehn Prozent. Diese Dimensionen, die ausschlaggebend für die Gefahr einer Staatspleite sind, geraten bei der von der BRD geforderten und von der EU verordneten „Konsolidierungspolitik“ nicht in den Blick.

Das den Griechen verordnete Sparpaket stammt aus der Folterkammer des Neoliberalismus. Gefordert wird die Senkung der Gehälter nicht nur der öffentlich Bediensteten, sondern allgemeiner „structural measures and wage moderation“, weitere Maßnahmen, um Flexicurity auf dem Arbeitsmarkt herzustellen, womit in guter Orwellscher Tradition versucht wird, die Entrechtung der abhängig Beschäftigten durch die Deregulierung des Arbeitsrechts mit dem Begriff Sicherheit zu verbinden. Gefordert wird eine Änderung, d.h. im Zweifel Privatisierung des Renten- und Gesundheitssystems und die Erhöhung des Rentenalters. Angeprangert wird von der Kommission, dass Griechenland weiter Wettbewerbsschranken aufrecht erhalte und insbesondere die Netze nicht liberalisiert seien. Wörtlich: „Third, the liberalization of network industries (e.g. energy) is lagging behind the EU average, as well as the opening of markets in the transport sector, especially in rail. For example, in the gas sector, the first private LNG (liquid natural gas) import was cancelled (December 2009) due to State-controlled grid operator DESFA failing to agree until then contracts providing access to network." Folglich sollen die Netze dem Wettbewerb geöffnet werden. ${ }^{14}$

$\mathrm{Ob}$ solche Maßnahmen zu einer Konsolidierung des griechischen Haushalts führen, ist eher fraglich. Ein anderer Effekt zeichnet sich aber bereits ab: Die Folgen der Umsteuerung auf einen rigiden Sparkurs sind in Griechenland am Einkaufsmanagerindex ${ }^{15}$ abzulesen. Dieser weist im Unterschied zu anderen Industriestaaten, für die seit Januar 2009 eine stetige Verbesserung zu beobachten ist, in Griechenland eine sinkende Tendenz auf. ${ }^{16}$ Das heißt, anders als Deutschland, das auf einen konjunkturellen Aufschwung hofft, ist in Griechenland auch für 2010 nicht zu erwarten, dass der „Konjunkturmotor“ anspringt, möglicherweise steht sogar ein Minus beim BIP an. Der Sparkurs verhindert das Wirtschaftswachstum und damit notwenig auch den Erfolg des Sparens, da die Steuereinnahmen eben auch nicht wachsen oder gar sinken. Nicht nur Griechenland, sondern Europa steht so vor einem zweiten Dilemma: Eine staatlich gestützte Ankurbelung der Konjunktur bei niedrigem Zinssatz und Arbeitszeitverkürzung

12 J. Bischoff, Der Euro, Griechenland und die zweite Phase der großen Krise, in: Sozialismus 6/2010, S. $19 \mathrm{ff}$.

13 FTD 27.4.2010.

14 Council Decision making public Council Recommendation No 6146/10 of 16 February 2010 with a view to ending the inconsistency with the broad guidelines of the economic policies in Greece and removing the risk of jeopardising the proper functioning of the economic and monetary union, http:// register.consilium.europa.eu/pdf/en/10/st06/st06146.en10.pdf; Recommendation for a Council Decision, Giving notice to Greece to take measures for the deficit reduction judged necessary in order to remedy the situation of excessive deficit, http://ec.europa.eu/economy_finance/sgp/pdf/30_edps/ 104-09_commission/2010-02-03_el_126-9_commission_en.pdf, S.5.

15 Der Einkaufsmanagerindex ist ein Konjunkturbarometer, entwickelt auf Basis der Befragung einer relevanten Auswahl von Einkaufsmanagern nach der Entwicklung von festgelegten Kenngrößen.

16 FTD vom 2.6.2010, S. 14 
bringt die Wirtschaft möglicherweise in Schwung - die USA fordern insbesondere von Deutschland, auf diese Linie einzuschwenken -, könnte aber die Staatsverschuldung weiter nach oben treiben. Eine Politik der Haushaltskonsolidierung könnte zur Senkung der Verschuldensquoten beitragen, ist aber sicher kontraproduktiv für die konjunkturelle Entwicklung.

\section{Faktisches und Widerrechtliches in der Transferunion}

Die strukturell problematische Differenz der Produktivität im Euro-Raum, die sich u.a. am Auseinanderfallen der Leistungsbilanzen ablesen lässt, wird von der Bundesregierung als Problem systematisch außer Betracht gelassen. So findet sich kein Weg zu europäischen Maßnahmen einer Gegensteuerung in Richtung einer Angleichung der Handelsströme. Deutschland und die Niederlande haben überdurchschnittliche Positiv-Salden in der Leistungsbilanz, während Portugal, Griechenland, Italien und Spanien, die als potenzielle Kandidaten einer Staatspleite betrachtet werden, deutlich im negativen Bereich liegen. Deutschland hat jahrelang eine Politik des „beggar thy neighbour“ betrieben: Durch niedrige Löhne konnten die Lohnstückkosten im Vergleich zu den Nachbarn gesenkt werden. Während in Deutschland der Binnenmarkt stagniert, konnten die Exporte in die europäischen Nachbarländer gesteigert werden, umgekehrt schrumpften die Importe relational. Ein solches Ungleichgewicht kann - wie man am Beispiel Ost- und Westdeutschland, in dem der eine Teil vorwiegend Markt ist, während im anderen konsumiert wird, sehen kann - nur bestehen, wenn gleichzeitig Transferleistungen bezahlt werden. ${ }^{17}$

Gegen Transferleistungen in Form des Stabilisierungsmechanismus hat sich die Bundesregierung vehement gewehrt und darauf verwiesen, dass die EU nach den Verträgen keine Transferunion sei. Das ist selbstverständlich nur bedingt richtig. Die Strukturfonds der EU bergen Ansätze einer Transferunion, wenn die Nettozahler auch mit Argusaugen darüber wachen, dass sie eine möglichst große Summe aus den Fonds zurück erhalten. Dem Umfang nach sind diese Fonds aber zu vernachlässigen, wenn man sie mit dem zentralen Mechanismus der europäischen Transferleistungen vergleicht: Auf den Agrarhaushalt entfielen traditionell bis zu zwei Drittel des Gesamthaushaltes der Union, was in der jüngeren Zeit auf „nur noch“ knapp $50 \%$ reduziert wurde. ${ }^{18}$ Das stützt einerseits die Agrarwirtschaft - auch gegen die Konkurrenz aus dem globalen Süden. Andererseits hat es aber als nicht-intendierten Effekt strukturkonservative Wirkungen, welche die beschworene Konkurrenzfähigkeit eher senkt. So arbeiten in Deutschland zwei, in den Niederlanden drei und in Frankreich knapp vier Prozent der Beschäftigten im primären Sektor. In Portugal und Griechenland sind es dagegen noch zwischen elf und zwölf Prozent der Beschäftigten, die in der Landwirtschaft arbeiten. ${ }^{19}$ So wird durch den finanziellen Transfer das strukturelle Ungleichgewicht möglicherweise verschärft und nicht ausgeglichen. Für eine Angleichung bedürfte es einer europäischen Wirtschaftsregierung, die sich an eine Struktur- und Rahmenplanung wagt und nicht ausschließlich in Kategorein von Preisstabilität und Gleichgewicht der Märkte denkt.

Der Abwehr der Transferunion bezog sich auf die „bail out“ Klausel in Art. 125 AEUV. Diese Vorschrift schließt zunächst einen bundesstaatlichen Finanzaus-

17 L. Zeise, Die Niederlage Berlins, in: Junge Welt v. 19.5.2010, S. 11.

18 Council of the European Union, 9596/10 (Presse 108) Press Release Extraordinary Council meeting Economic and Financial Affairs Brussels, 9/10 May 2010, http://ec.europa.eu/publications/archives/ booklets/move/13/txt_de.htm.

19 Bundeszentrale für politische Bildung (http://www.bpb.de/themen/17ISON,0,0,Mitgliedstaaten.html). 
gleich oder Finanztransfers in Haushaltsnotlagen, wie sie in Deutschland verfassungsrechtlich geboten sind, aus. Der "Stabilisierungsmechanismus“ wurde auf eine andere Grundlage gestützt: Der neue Europäische Stabilitätsmechanismus, heißt es in der Erklärung des ECOFIN, „is based on Art. 122.2 of the Treaty and an intergovernmental agreement of euro area Member States“. ${ }^{20}$ Der zweite Teil macht den tatsächlichen Charakter der Vereinbarung deutlich: Es handelt sich um eine zwischenstaatliche Vereinbarung der Euro-Staaten, die also neben dem Lissaboner Vertrag herläuft. Wenn der Stabilitätsmechanismus auf eine intergovernmentale Vereinbarung gestützt werden muss, wird die Unzulänglichkeit der Europäischen Verträge geradezu offensichtlich. Die Währungsunion ist als Schön-Wetter-Konstrukt erdacht, das auf der irrigen Annahme eines natürlichen Gleichgewichts der Märkte basiert.

Der zweite Teil des Rettungspaketes besteht darin, dass die EZB (griechische) Staatsanleihen aufkauft. Der EZB-Rat hat beschlossen, „Interventionen an den Märkten für öffentliche und private Schuldverschreibungen im Euro-Währungsgebiet (Programm für die Wertpapiermärkte) durchzuführen. “21 Das steht in direktem Widerspruch zu Art. 123 Abs. 1 AEUV, wonach Überziehungs- oder andere Kreditfazilitäten bei der Europäischen Zentralbank für die Mitgliedstaaten ebenso verboten sind wie der unmittelbare Erwerb von Schuldtiteln von diesen durch die Europäische Zentralbank. Diese Vorschrift ist - wie sich jetzt zeigt - nicht nur finanzpolitischer Unsinn, weil der Zentralbank in der Krise Interventionsmöglichkeiten genommen werden. Sie ist gleichzeitig eine Gewinngarantie für die Banken, die bei der EZB zu niedrigen Zinsen Geld leihen können, um es zu hohen Zinsen den Staaten (Griechenland) zu leihen.

Weil von den Maastricht-Kriterien und vom Stabilitäts- und Wachstumspakt die Gesamtverschuldensgrenze mehr oder weniger unter den Tisch gefallen ist und auch von der Kommission nur noch die $3 \%$-Grenze - dafür um so intensiver, aber mit ungewissem Ausgang - verfolgt wird, hat sich der Lissabon-Vertrag schon wenige Monate nach seinem Inkrafttreten als fahruntüchtiges Vehikel erwiesen, jedenfalls wenn es in einen Sturm gerät.

\section{Politische Union und Wirtschaftsregierung}

Das scheinen auch zumindest Teile der Bundesregierung so zu sehen. In den „Eckpunkten der Bundesregierung zur Stärkung der Eurozone“ vom Mai 2010 heißt es unmissverständlich: „Wenn wir einen dauerhaft tragfähigen Rahmen für die Währungsunion anstreben, müssen wir auch die Möglichkeit von Vertragsänderungen in unsere Überlegungen mit einbeziehen. “22 Die Eckpunkte gehen etwas weiter als die Vorschläge der Kommission zur Stabilisierung der EuroZone, ${ }^{23}$ zielen aber in die gleiche Richtung. Die Dosis der wirkungslosen und z.T. unbekömmlichen Medizin soll verstärkt werden. Die Vorschläge zielen auf eine Effektivierung des „Stabilitäts- und Wachstumspaktes“, d.h. auf eine zen-

20 Council of the European Union, 9596/10 (Presse 108) Press Release Extraordinary Council meeting Economic and Financial Affairs Brussels, 9/10 May 2010, http://www.consilium.europa.eu/uedocs/ cms_data/docs/pressdata/en/ecofin/114324.pdf.

21 EZB, Pressemitteilung vom 10. Mai 2010, http://www.bundesbank.de/download/ezb/pressenotizen/ 2010/20100510.ezb.pdf.

22 BMF, Eckpunkte der Bundesregierung zur Stärkung der Eurozone, http://www.bundesfinanzministerium.de/nn_54/DE/Wirtschaft_und_Verwaltung/Europa/Der_Euro/20100520-Task-Force.html? - nnn=true.

23 Communication (12.5.2010) from the commission to the European Parliament, the European Council, the Council, the European Central Bank, the Economic and Social Committee and the Committee of the Regions, Reinforcing economic policy coordination, http://eur-lex.europa.eu/LexUriServ/ LexUriServ.do?uri=COM:2010:0250:FIN:EN:PDF. 
trale Überwachung und verschärfte Sanktionen. Kommission und Bundesfinanzminister schlagen vor, die nationalen Haushalte vor ihrer Verabschiedung durch die nationalen Parlamente auf ihre Verträglichkeit mit den Defizitkriterien und den wirtschaftspolitischen Zielen der EU überprüfen zu lassen.

Dabei sieht das BMF das Problem der demokratischen Legitimation durchaus. Wenn die EU, wie es das Bundesverfassungsgericht im Lissabon-Urteil24 feststellte, sich (noch) nicht selbst demokratisch legitimieren kann, ihre Entscheidungen vielmehr mittelbar über die nationalen Parlamente legitimiert werden müssen, dann ist es höchst problematisch, die nationalen Parlamente ausgerechnet in ihrem Budgetrecht zu beschränken, indem man dieses unter europäische Aufsicht stellt. Die Lösung des BMF ist keine: „Daher wäre es ein logischer Schritt, wenn unsere nationalen Parlamente stärker in die europäische Finanzpolitik eingebunden werden. So könnten die Euro-Finanzminister in ihren nationalen Parlamenten Rechenschaft ablegen über die Prüfung der Stabilitätsprogramme ihrer Partner in der Eurozone. Damit könnte die nationale Verantwort-

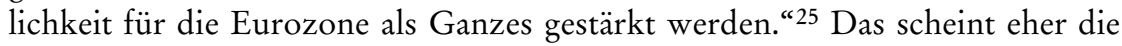
perfide Formulierung für den Tatbestand zu sein, dass der deutsche Bundestag nicht nur gleichsam den deutschen Anteil der EU legitimiert, sondern eventuell auch noch den griechischen, wenn dort gegen die Defizitkriterien verstoßen wird. Die Finanz- und Wirtschaftskrise hat auch in ihrem zweiten Höhepunkt, der Griechenlandkrise, gezeigt, dass eine Neujustierung der Europäischen Politik überfällig ist. Merkels Abwehr einer Wirtschaftsregierung, wie sie als ersten Ansatz Sarkozy vorgeschlagen hat, durch ihre Erweiterung auf alle 27 EU Staaten, frönt weiter dem Marktglauben und dürfte die Krise des Euro und der EU weiter verschärfen. Georg Soros bemerkt im oben zitierten Artikel: „Der Euro ist eine unvollständige Währung: Der Vertrag von Maastricht begründet eine Währungsunion ohne politische Union - mit Zentralbank, aber ohne zentrales Finanzministerium. “26 Die europäische Union hat den Nationalstaaten die Steuerungsmedien in der Wirtschaftspolitik aus der Hand geschlagen, selber aber auf Steuerungskompetenzen durch Zins-, Steuer- und Haushaltspolitik verzichtet in dem irrigen Glauben, der Markt werde es schon richten. Für die Wiederaneignung des Primats der Politik in der EU bleibt möglicherweise nur wenig Zeit - bis zum nächsten Akt der großen Krise der kapitalistischen Weltwirtschaft. ische Demokratie ein!, Kritische Justiz, Heft 3/2009, S. 220.

25 BMF, Eckpunkte der Bundesregierung zur Stärkung der Eurozone, http://www.bundesfinanzministerium.de/nn_54/DE/Wirtschaft_und_Verwaltung/Europa/Der_Euro/20100520-Task-Force.html? - nnn=true. 